\title{
FMRP and its target RNAs: fishing for the specificity
}

\author{
Massimiliano Veneri,' Francesca Zalfa, ${ }^{1,2}$ and Claudia Bagni,2,CA \\ 'Dipartimento di Biologia, Università di Roma Tor Vergata, Via della Ricerca Scientifica I, Roma; ${ }^{2}$ Istituto di Neuroscienze Sperimentali, \\ Fondazione Santa Lucia, IRCCS. Via Ardeatina, 306, Roma, Italy \\ CACorresponding author: claudia.bagni@uniroma2.it
}

Received 30 July 2004; accepted 9 September 2004

Learning and memory difficulties observed in patients with fragile $X$ syndrome, as well as in a mouse model for the syndrome, are partially due to impaired translational regulation of neuronal mRNAs encoding key molecules for the synaptic structure and function. There has been intense interest in characterizing the mRNAs that are regulated by the fragile $X$ mental retardation protein (FMRP) in the neuronal cell. A large number of candidate
FMRP-interacting mRNAs has been identified over the last few years and three models have been described so far to explain the specificity of these interactions. Here, we report our vision on how they could work in the same and/or in different pathways and suggest that the three mechanisms may not be mutually exclusive. NeuroReport 15:2447-2450 (C) 2004 Lippincott Williams \& Wilkins.

Key words: Fragile X mental retardation protein; G-quartet; BCl; Micro RNAs; Translational regulation

\section{INTRODUCTION}

The fragile $X$ mental retardation protein (FMRP) is an RNAbinding protein mostly localized in the cytoplasm. It contains both a nuclear localization signal (NLS) and a nuclear export signal (NES) allowing it to shuttle between nucleus and cytoplasm $[1,2]$.

Absence or reduction of FMRP leads to fragile $X$ syndrome (FXS), the most common form of inherited mental retardation, with an estimated prevalence of 1/4000 males and 1/6000 females. The syndrome is characterized clinically by notable deficits in language and executive functions. Patients often manifest attention-deficit, hyperactivity disorder and autistic-like behaviour [3]. In addition FXS is also associated with a specific set of physical manifestations, including distinctive facial features, connective tissue abnormalities, and macroorchidism in males [4,5]. One of the most relevant characteristics is mental retardation [6]. Morphological studies on post-mortem patient brains showed the presence of abnormal dendritic spines $[7,8]$. Moreover the mouse model for the syndrome (FMRP knockout) [9] has the same spine dysmorphogenesis $[10,11]$. This very likely reflects a dysregulation of molecular events underlying the synaptic structure, such as mRNA transport/localization or local protein synthesis.

Functional analysis and comparison of the sequence domains of FMRP shows the presence of four RNA-binding motifs: two ribonucleoprotein $\mathrm{K}$ homology domains $(\mathrm{KH}$ domains), a cluster of arginine and glycine residues (RGG box), and a novel RNA-binding domain present in the Nterminal region of the protein [12-15]. FMRP binds to RNA homopolymers in vitro $[12,14]$ and also to $4 \%$ of total human fetal brain mRNAs in vitro [16]. These latter findings suggested that FMRP could have some RNA-binding selectivity. Several groups have taken different approaches in order to identify the in vivo mRNA targets for FMRP and the RNA elements required for FMRP-RNA interaction. There are at least two categories of mRNAs recognized by FMRP (for a recent review see [17]). The first includes mRNAs with a structure called the G-quartet [18-20] while the second includes mRNAs complementary and bound to the non coding RNA BC1 [22].

Brown et al. [23] examined the mRNAs associated with FMRP-mRNP in mouse brain and human lymphoblastoid cell lines. From this and parallel studies [18,19] an interesting RNA structure recognized by FMRP in vivo has been identified and characterized. In particular, it has been shown that FMRP is able to bind mRNAs containing a series of guanine that can be arranged in a G-quartet. G-quartets are nucleic acid structures in which four guanine residues are arranged in a planar conformation stabilized by Hoogsteen-type hydrogen bonds. Some of the FMRP-target mRNAs including its own mRNA [18] have a G-quartet motif but the majority does not, leaving open the question of how the rest of the mRNAs are recognized. To better understand the FMRP function in neurons Eberwine and Greenough developed and used a novel technology for the identification of mRNA targets called APRA (antibody positioned RNA amplification). This method utilizes for the priming of cDNA synthesis oligonucleotides linked to antibodies against FMRP. New molecules were isolated and some contained a G-quartet motif [24].

Finally, we showed that translation of specific neuronal mRNAs is up-regulated in FMR1 knockout mice. Significantly, the translational repression by FMRP is much stronger in isolated synaptoneurosomes than in total brain extracts. In addition, we have shown that FMRP binds to the small non-messenger cytoplasmic RNA BC1, and $\mathrm{BC} 1$ simultaneously associates with mRNA targets regulated by FMRP [22]. These data suggest that $\mathrm{BC} 1$ could mediate the interaction between FMRP and the regulated mRNAs. 


\section{HOW DOES FMRP RECOGNIZE ITS SPECIFIC TARGET mRNAs?}

FMRP can bind directly to mRNAs: While the high affinity that FMRP has for RNA molecules in general is extremely clear from many papers published over the last ten years, it is now possible to identify three putative mechanisms that allow FMRP to specifically recognize its mRNA targets. The first significative study shedding light on the FMRP-RNA interaction came from Brown et al. [23]. They co-immunoprecipitated mRNAs with the FMRP ribonucleoprotein complex and used these mRNAs to screen microarrays. They identified one set that encompasses $432 \mathrm{mRNAs}$ associated with FMRP in the mouse brain. In parallel studies, by comparing the mRNA polysomal profile from normal and fragile $X$ patient lymphoblastoid cells, they found another set of 251 mRNAs, which had variations in their distribution on polysome gradients while their cytoplasmic abundance remained unchanged. The two groups have 14 mRNAs in common, which encode proteins important for the neuronal function, synaptic plasticity and neuronal maturation [23] and interestingly, among them, they also include the microtubule associated protein 1B (MAP1B) mRNA that has also been found to be translationally regulated in Drosophila mutants for the homologous FMR1 gene $[22,25]$.

Darnell et al. [19] have shown, using in vitro selection of random RNA sequences (SELEX) that FMRP binds a class of mRNAs with G-quartet motifs. Of 31 candidate mRNAs containing this motif identified in the database, 12 were assayed for FMRP binding and six RNAs bound to FMRP with considerable affinities. All of them are associated with synaptic function and also included MAP1B mRNA. This joined work thus yielded a list of 6 RNA targets containing $\mathrm{G}$ quartets that directly bind to FMRP and change their translational efficiency in fragile $X$ cell lines [19].

Finally, using the sophisticated APRA technique, 81 additional mRNAs were identified by Miyashiro et al. which encode proteins with diverse physiological properties such as cytoskeleton structure and function, synaptic transmission, peroxisomal biogenesis, membrane docking and fusion, nuclear transport and molecular chaperone-like activity [24]. While there is not much overlap with the mRNAs identified in previous studies, some mRNAs $(23 \%)$ contain the G-quartet structure confirming and extending previously findings $[18,19]$. We can thus envisage a first molecular mechanism of FMRP binding through the recognition of particular RNA sequences able to adopt a G-quartet structure (Fig. 1a). Domain mapping has shown that the RGG box of FMRP binds the G-quartet motifs $[18,19]$. This is quite interesting because it brings a new vision into the function of the arginine/glycine-rich domains which are generally believed to promote non-specific binding to nucleic acids through electrostatic interactions and are also considered to have the property to unfold RNA secondary structures $[26,27]$. A great degree of heterogeneity in the conformation of the RNA targets and in their RGG binding mode has been recently reported by Pastore's team [20], suggesting that other cis-acting sequences in target mRNAs may also be involved in their association with FMRP. Indeed, recently new FMRP targets have been isolated containing U-rich stretches [21]. The combined action of the RGG box and other RNA binding domains could thus direct FMRP to mRNAs that have G-rich and U-rich elements in vicinity.
BC1 can function as an adapter between FMRP and its target mRNAs: The second model that could explain the recognition of other mRNAs would be through the association with the small dendritic non-coding RNA BC1, which anneals to complementary regions of some mRNA targets, thereby recruiting FMRP to the targeted mRNAs and determining the specificity of FMRP action [22] (Fig. 1b). In particular we found that $\mathrm{BC} 1 \mathrm{RNA}$ is predicted to basepair to the neuronal mRNAs encoding for molecules important for the synaptic structure and function (MAP1B mRNA appears to be among these), with a sequence complementarity found at the base of the longer stem-loop, according to a stable predicted secondary structure of BC1 RNA [28]. A recent study from Darlix's laboratory [29] supports the hypothesis that the recognition mechanism is mediated by the association of FMRP with BC1 RNA. Moreover, they found that FMRP possesses all the properties of a potent nucleic acid chaperone which promotes the annealing of nucleic acids with complementary sequences as well as strand exchange in a duplex nucleic acid structure in vitro [29], raising the possibility that FMRP plays a direct role in $\mathrm{BC} 1 / \mathrm{mRNA}$ annealing.

Thus, BC1 is an important factor in FMRP-mediated translational repression, but there is still an interesting question to answer: which is the RNA binding domain responsible for the binding? The RGG box has been involved in the G-quartet recognition and BC1 does not contain an obvious G-quartet or a U-rich sequence. This means that $\mathrm{BC} 1$ could bind to one of the other three RNAbinding domains. Infact, we have recently found that the Nterminus of FMRP, which exhibits no homology to known RNA binding domains, binds strongly and specifically to the $5^{\prime}$ stem-loop of BC1 (unpublished data), this is also the region which exhibits complementarity to FMRP target mRNAs. The interaction of FMRP with BC1 RNA may also give a clue at how FMRP regulates translation. In fact the interaction with $\mathrm{BC} 1$ could mediate the associations of ribonucleocomplex containing FMRP with some components of the preinitiation complex [30]. In support of this notion, $\mathrm{BC} 1$ has also been shown to associate with poly(A) binding protein and eIF4A $[30,31]$. Thus, the BC1/mRNA/ FMRP complex may form the translational inhibition complex (Fig. 1b).

The molecular mechanisms so far described could actually work synergistically. Where a weakly conserved purine quartet, a motif containing few Us, or a region with comparatively low complementarity to $\mathrm{BC} 1$ each may not suffice to bind FMRP, several such elements in vicinity may be more efficient. MAP1B mRNA is a typical example, it has been found to be associated to FMRP using independent approaches and presents both a G-quartet and a long complementary region to $\mathrm{BC} 1$.

Interestingly, the two $\mathrm{KH}$ domains of FMRP, which seem to recognize RNA in a sequence-specific way are still orphans for targets except for a single report were the authors identified as bound to the $\mathrm{KH} 2$ domain mainly repetitive elements [32].

FMRP and miRNAs: A third mechanism is suggested by a recently described RNP complex that involves the Drosophila ortholog dFXR and some components of the RISC (RNA-induced silencing complex) such as the proteins AGO2, DmP68, VIG and micro-RNAs [33-35]. Micro-RNAs 
(a)

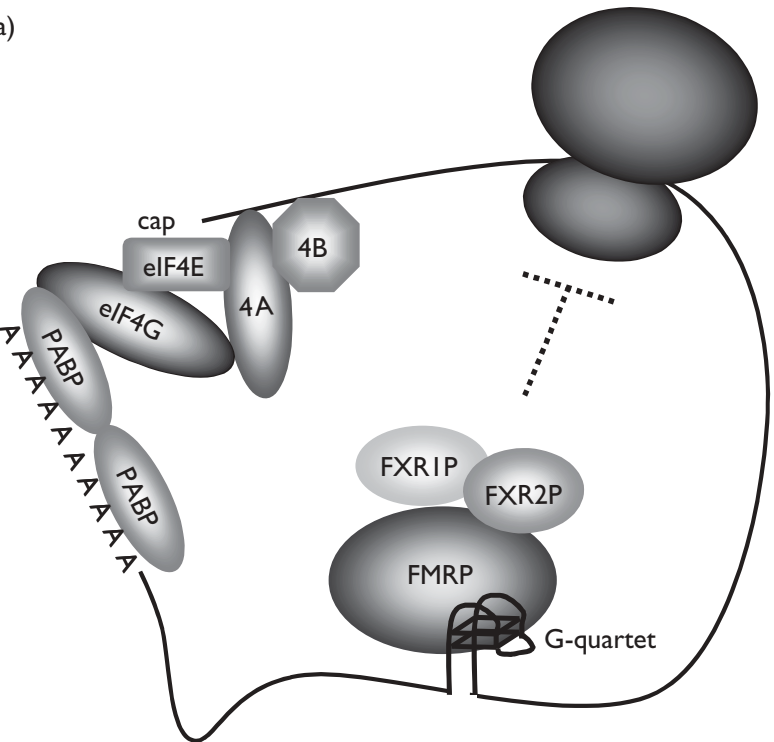

(b)

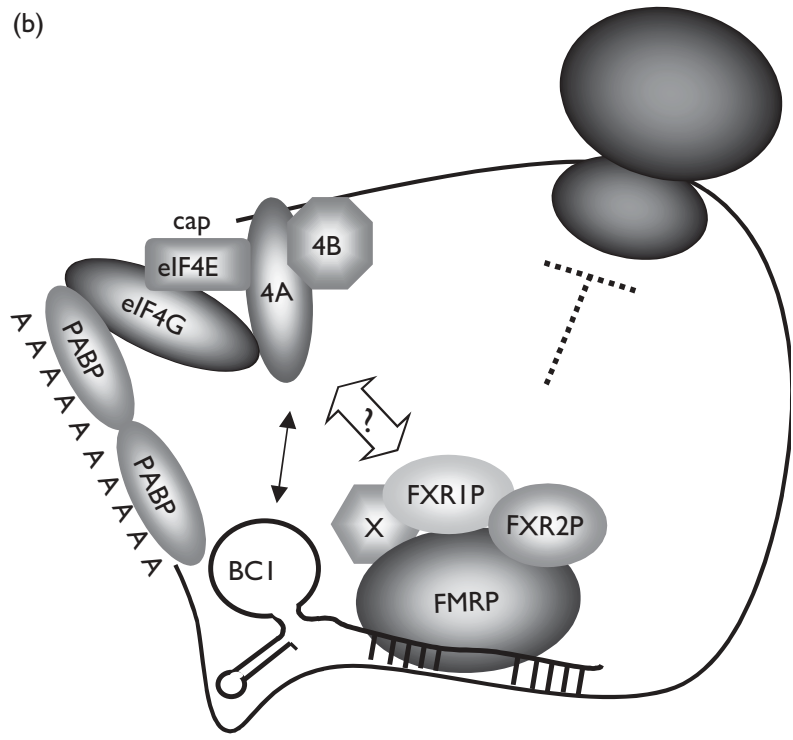

(c)

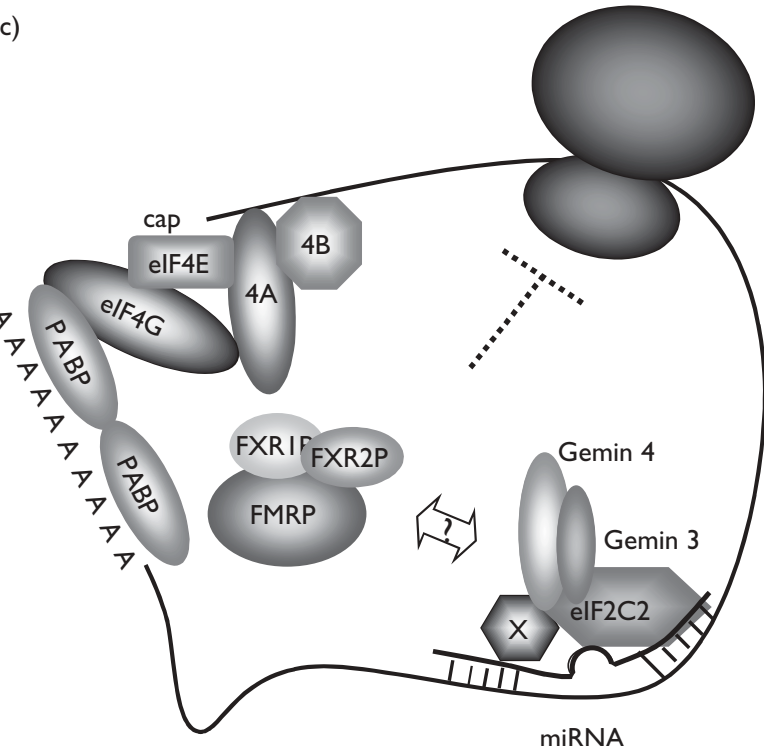

(miRNAs) are a large family of short (20-24 nucleotides) single-stranded, non-coding regulatory RNAs that modulate gene expression by partially base-pairing with target mRNA sequences. Micro-RNAs are derived from the processing of larger precursor of $\sim 70$ nucleotides or longer by the endonuclease Dicer (for a recent review see [36]).

Because the final goal of FMRP action is the translational regulation of a subset of neuronal mRNAs, it is indeed important that several components of the miRNA pathway have been implicated in translational control, including Gemin3, Gemin4 and eIF2C2, a member of the human Argonaute family [37]. What is the biological meaning of the association of FMRP with the putative RISC? FMRP could be targeted to its mRNA targets as part of a RISC complex and then, with or without binding directly its mRNA targets, could regulate (repress) translation guided by miRNAs [33-35] (Fig. 1c). Indeed recently it has been shown that human FMRP is associated to miRNAs [38]. FMRP could play a part in this regulatory pathway, stabilizing the specific annealing between miRNAs and the complementary region in $3^{\prime}$-UTR of the target mRNAs. Finally, BC1 structure reminds very much a miRNA precursor strengthening the real possibility that FMRP is attracted by double-stranded mRNA to mediate the specific recognition. These results strongly support the hypothesis that FMRP can act to stabilize the RNA-RNA duplex which must have been formed between $\mathrm{BC} 1$ and the mRNA targets or between miRNAs and the complementary sequence in the $3^{\prime}$-UTR of substrate transcripts (Fig. 1b,c). From these recent data, it seems that both $\mathrm{BC} 1$ and miRNAs have similar mechanisms of action.

Comparison of the models presented in this review poses the compelling question of whether the models are in conflict with each other. We propose that they are perfectly compatible with each other, since at least BC1 RNA and the G-quartet motif bind to distinct domains on the FMRP protein. Integration of these three models may explain the complexity of the syndrome and the variability that is observed in patients with the same FMRP deficiency. Furthermore, it is even possible that other mechanisms may be still involved that would require the functional activity of the two $\mathrm{KH}$ domains. It should be noted that often the binding-specificity of a multi-domain RNAbinding protein is different from the sum of the binding specificities of the individual domains.

The mode of RNA recognition could also be regulated during development. Indeed, for the last mechanism involving miRNAs it is well established that it works mainly during development [39-42]. In adulthood, when the FMRP protein is still necessary for the brain function, other mechanisms could take over.

Whatever the mechanism of RNA recognition with FMRP, loss of FMRP in affected individuals leads to impaired expression (mRNA localization? and/or mRNA translation) of a subset of neuronal mRNA. A more detailed analysis of

Fig. I. Three possible models for FMRP function. (a) FMRP recognizes its mRNA targets containing the G-quartet structure and this could lead to translational repression. (b) FMRP represses mRNA translation via $\mathrm{BCl}$ RNA interaction. In this case $\mathrm{BCl}$ could mediate the translation inhibition by the association with some components of the preinitiation complex such as the poly $(\mathrm{A})$ binding protein and the elF4A. (c) FMRP can repress mRNA translation via miRNAs. The FMRP-RISC complex would recognize the target mRNAs via miRNAs and repress mRNA translation. 
the mRNAs isolated so far should help to unravel the fine molecular events surrounding the syndrome as well as the multitask functions of FMRP.

\section{REFERENCES}

1. Eberhart DE, Malter HE, Feng $Y$ and Warren ST. The fragile $X$ mental retardation protein is a ribonucleoprotein containing both nuclear localization and nuclear export signals. Hum Mol Genet 1996; 5:1083-1091.

2. Bardoni B, Sittler A, Shen Y and Mandel JL. Analysis of domains affecting intracellular localization of the FMRP protein. Neurobiol Dis 1997; 4: 329-336.

3. Hagerman RJ and Cronister A. Fragile X Syndrome Diagnosis, Treatment, and Research. Baltimore, MD: Johns Hopkins University Press; 1996, pp. 3-87.

4. O'Donnell WT and Warren ST. A decade of molecular studies of fragile X syndrome. Annu Rev Neurosci 2002; 25:315-338.

5. Slegtenhorst-Eegdeman KE, de Rooij DG, Verhoef-Post M, van de Kant HJ, Bakker CE, Oostra BA et al. Macroorchidism in FMR1 knockout mice is caused by increased Sertoli cell proliferation during testicular development. Endocrinology 1998; 139:156-162.

6. Chiurazzi P, Neri G and Oostra BA. Understanding the biological underpinnings of fragile X syndrome. Curr Opin Pediatr 2003; 15:559-566.

7. Hinton VJ, Brown WT, Wisniewski K and Rudelli RD. Analysis of neocortex in three males with the fragile $\mathrm{X}$ syndrome. Am J Med Genet 1991; 41:289-294.

8. Irwin SA, Galvez R and Greenough WT. Dendritic spine structural anomalies in fragile-X mental retardation syndrome. Cerebr Cortex 2000; 10:1038-1044.

9. Bakker CE, Verheij C, Willemsen R, van der Helm R, Oerlemans F, Vermey $\mathrm{M}$ et al. Fmr1 knockout mice: a model to study fragile $\mathrm{X}$ mental retardation. The Dutch-Belgian Fragile X Consortium. Cell 1994; 78:23-33.

10. Comery TA, Harris JB, Willems PJ, Oostra BA, Irwin SA, Weiler IJ et al. Abnormal dendritic spines in fragile $X$ knockout mice: maturation and pruning deficits. Proc Natl Acad Sci USA 1997; 94:5401-5404.

11. Braun $\mathrm{K}$ and Segal M. FMRP involvement in formation of synapses among cultured hippocampal neurons. Cerebr Cortex 2000; 10:1045-1052.

12. Siomi H, Matunis MJ, Michael WM and Dreyfuss G. The pre-mRNA binding $\mathrm{K}$ protein contains a novel evolutionarily conserved motif. Nucleic Acids Res 1993; 21:1193-1198.

13. Siomi M, Siomi H, Sauer WH, Srinivasan S, Nussbaum RL and Dreyfuss G. FXR1, an autosomal homolog of the fragile $X$ mental retardation gene. EMBO J 1995; 14:2401-2408.

14. Adinolfi S, Bagni C, Musco G, Gibson T, Mazzarella L and Pastore A. Dissecting FMR1, the protein responsible for fragile $\mathrm{X}$ syndrome, in its structural and functional domains. RNA 1999; 9:1248-1258.

15. Adinolfi S, Ramos A, Martin SR, Dal Piaz F, Pucci P, Bardoni B et al. The $\mathrm{N}$-terminus of the fragile $\mathrm{X}$ mental retardation protein contains a novel domain involved in dimerization and RNA-binding. Biochemistry 2003; 42:10437-10444.

16. Ashley CT, Jr. Wilkinson KD, Reines D and Warren ST. FMR1 protein: conserved RNP family domains and selective RNA binding. Science 1993; 262:563-566.

17. Zalfa F and Bagni C. Molecular insights into mental retardation: multiple functions for the fragile X mental retardation protein? Curr Issues Mol Biol 2004; 6:73-88

18. Schaeffer C, Bardoni B, Mandel JL, Ehresmann B, Ehresmann C and Moine $\mathrm{H}$. The fragile $\mathrm{X}$ mental retardation protein binds specifically to its mRNA via a purine quartet motif. EMBO J 2001; 20:4803-4813.

19. Darnell JC, Jensen KB, Jin P, Brown V, Warren ST and Darnell RB. Fragile $X$ mental retardation protein targets $G$ quartet mRNAs important for neuronal function. Cell 2001; 107:489-499.

20. Ramos A, Hollingworth D and Pastore A. G-quartet-dependent recognition between the FMRP RGG box and RNA. RNA 2003; 9:1198-1207.

21. Chen L, Yun SW, Seto J, Liu $W$ and Toth $M$. The fragile $X$ mental retardation protein binds and regulates a novel class of mRNAs containing U rich target sequences. Neuroscience 2003; 120:1005-1017.
22. Zalfa F, Giorgi M, Primerano B, Moro A, Di Penta A, Reis S et al. The fragile $X$ syndrome protein FMRP associates with BC1 RNA and regulates the translation of specific mRNAs at synapses. Cell 2003; 112:317-327.

23. Brown V, Jin P, Ceman S, Darnell JC, O'Donnell WT, Tenenbaum SA et al. Microarray identification of FMRP-associated brain mRNAs and altered mRNA translational profiles in fragile X syndrome. Cell 2001; 107: 477-487

24. Miyashiro KY, Beckel-Mitchener A, Purk TP, Becker KG, Barret T, Liu L et al. RNA cargoes associating with FMRP reveal deficits in cellular functioning in Fmr1 null mice. Neuron 2003; 37: 417-431.

25. Zhang YQ, Bailey AM, Matthies HJ, Renden RB, Smith MA, Speese SD et al. Drosophila fragile X-related gene regulates the MAP1B homolog Futsch to control synaptic structure and function. Cell 2001; 107:591-603.

26. Kiledjian $M$ and Dreyfuss $G$. Primary structure the binding activity of the hnRNP U protein: binding through the RGG box. EMBO J 1992; 11: 2655-2664.

27. Dandekar T. RNA Motifs and Regulatory Elements. Springer, 2nd edn, 2002.

28. Rozhdestvensky TS, Kopylov AM, Brosius J and Huttenhofer A. Neuronal BC1 RNA structure: evolutionary conversion of a tRNA(Ala) domain into an extended stem-loop structure. RNA 2001; 7: 722-730.

29. Gabus C, Mazroui R, Tremblay S, Khandjian EW and Darlix JL. The fragile $X$ mental retardation protein has nucleic acid chaperone properties. Nucleic Acid Res 2004; 32:2129-2137.

30. Wang H, Iacoangeli A, Popp S, Muslimov IA, Imataka H, Sonenberg N et al. Dendritic BC1 RNA: functional role in regulation of translation initiation. J Neurosci 2002; 22:10232-10241.

31. Muddashetty R, Khanam T, Kondrashov A, Bundman M, Iacoangeli A, Kremerskothen $\mathrm{J}$ et al. Poly(A)-binding protein is associated with neuronal BC1 and BC200 ribonucleoprotein particles. J Mol Biol 2002; 321:433-445

32. Sung YJ, Conti J, Currie JR, Brown WT and Denman RB. RNAs that interact with the fragile $X$ syndrome RNA binding protein FMRP. Biochem Biophys Res Commun 2000; 275:973-980.

33. Carthew RW. RNA interference: the fragile $X$ syndrome connection. Curr Biol 2002 12:R852-854.

34. Caudy AA, Myers M, Hannon GJ and Hammond SM. Fragile X-related protein and VIG associate with the RNA interference machinery. Genes Dev 2002; 16:2491-2496.

35. Ishizuka A, Siomi MC and Siomi H. A Drosophila fragile X protein interacts with components of RNAi and ribosomal proteins. Genes Dev 2002; 16:2497-2508.

36. Bartel DP. MicroRNAs: genomics, biogenesis, mechanism, and function. Cell 2004; 116:281-297.

37. Nelson PT, Hatzigeorgiou AG and Mourelatos Z. miRNP:mRNA association in polyribosomes in a human neuronal cell line. RNA 2004; 10:387-394.

38. Jin P, Zarnescu DC, Ceman S, Nakamoto M, Mowrey J, Jongens TA et al. Biochemical and genetic interaction between the fragile $X$ mental retardation protein and the microRNA pathway. Nature Neurosci 2004; 7:113-117.

39. Lee RC, Feinbaum RL and Ambros V. The C. elegans heterochronic gene lin-4 encodes small RNAs with antisense complementarity to lin-14. Cell 1993; 75:843-854.

40. Wightman B, Ha I and Ruvkun G. Posttranscriptional regulation of the heterochronic gene lin-14 by lin-4 mediates temporal pattern formation in C. elegans. Cell 1993; 75:855-862.

41. Reinhart BJ, Slack FJ, Basson M, Pasquinelli AE, Bettinger JC et al. The 21-nucleotide let-7 RNA regulates developmental timing in Caenorhabditis elegans. Nature 2000; 403:901-906.

42. Brennecke J, Hipfner DR, Stark A, Russell RB and Cohen SM. Bantam encodes a developmentally regulated microRNA that controls cell proliferation and regulates the proapoptotic gene hid in Drosophila. Cell 2003; 113:25-36. 